\title{
\begin{tabular}{l} 
FREE \\
ACCESS \\
\hline
\end{tabular}
}

\section{Sizing-up nutrient uptake kinetics: combining a physiological trade-off with size-scaling of phytoplankton traits}

\author{
S. Lan Smith ${ }^{1,2, *}$, Agostino Merico ${ }^{3,4}$, Sönke Hohn ${ }^{3}$, Gunnar Brandt ${ }^{3}$ \\ ${ }^{1}$ Research Center for Global Change Research, JAMSTEC (Japan Agency for Marine-Earth Science and Technology), \\ 3173-25 Showa-machi, Kanazawa-ku, Yokohama 236-0001, Japan \\ ${ }^{2}$ CREST (Core Research for Evolutional Science and Technology), Japan Science and Technology Agency, Tokyo 102-0076, Japan \\ ${ }^{3}$ Systems Ecology, ZMT (Leibniz Center for Tropical Marine Ecology), Fahrenheitstrasse 6, 28359 Bremen, Germany \\ ${ }^{4}$ School of Engineering and Science, Jacobs University, Campus Ring 1, 28759 Bremen, Germany
}

\begin{abstract}
We present a new model for the kinetics of nutrient uptake by phytoplankton, which we derive by combining existing models: (1) a size-based modeling framework that accounts for the effects of extra-cellular diffusive limitation, and (2) a model of optimal resource allocation that maximizes uptake rate subject to a physiological trade-off. We show that the new model, unlike either of its pre-existing components, is able to reproduce the observed range of variation in the half-saturation constant for nitrate uptake as a function of both ambient nitrate concentration and cell size. Our results illustrate that the combination of mechanistic trait-based modeling and physiological trade-offs can greatly advance our understanding of plankton ecology.
\end{abstract}

KEY WORDS: Phytoplankton $\cdot$ Nutrient uptake kinetics $\cdot$ Size $\cdot$ Trait $\cdot$ Model $\cdot$ Trade-off

\section{INTRODUCTION}

Small phytoplankton species tend to dominate in low-nutrient regions of the ocean, whereas large species tend to thrive in nutrient-rich waters (Chisholm 1992, Marañón et al. 2001, Li 2002, Kostadinov et al. 2010). This is explained in terms of smaller species having higher surface area to volume ratios, higher affinities for nutrient uptake, lower diffusive limitation, and lower nutrient requirements relative to larger phytoplankton (Litchman \& Klausmeier 2008, Finkel et al. 2010). Allometric scaling relationships based on laboratory experiments confirm this general pattern (Litchman et al. 2007, Edwards et al. 2012). While satellite-based (e.g. Kostadinov et al. 2010) and ship-based observations (Marañón et al. 2001, Li 2002) reveal the combined effects of physiological acclimation, evolutionary adaptation, and shifts in species composition within communities, laboratory studies measure physiological acclimation and differ- ences between species under controlled conditions. Trait-based modeling aims to combine the information gained from field studies and laboratory experiments into a consistent framework of processes operating at vastly different scales (Edwards et al. 2013).

Theories and models have been developed to account for the size-scaling of physiological properties, including the effects of extra-cellular diffusive limitation (Armstrong 2008, Aksnes \& Cao 2011, Bonachela et al. 2011, Fiksen et al. 2013). Trade-offs in cellular nutrient allocation to different enzymes provide explanations for observed patterns of phytoplankton community structure (Litchman et al. 2007, Edwards et al. 2012), which have also been incorporated into models (Bruggeman \& Kooijman 2007, Ward et al. 2013, Wirtz 2013). Specifically for nutrient uptake physiology, Pahlow (2005) proposed a trade-off between affinity and maximum uptake rate to explain the physiological acclimation of phytoplankton in laboratory experiments (Fig. 1A). Smith et al. (2009) 


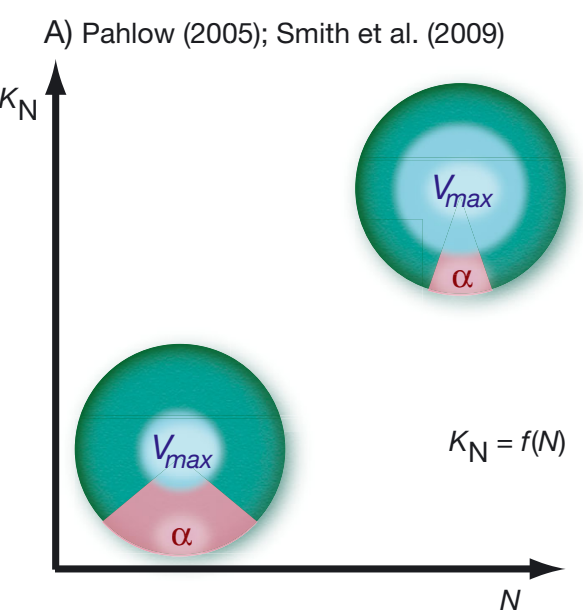

B) Aksnes \& Cao (2011); Fiksen et al. (2013)

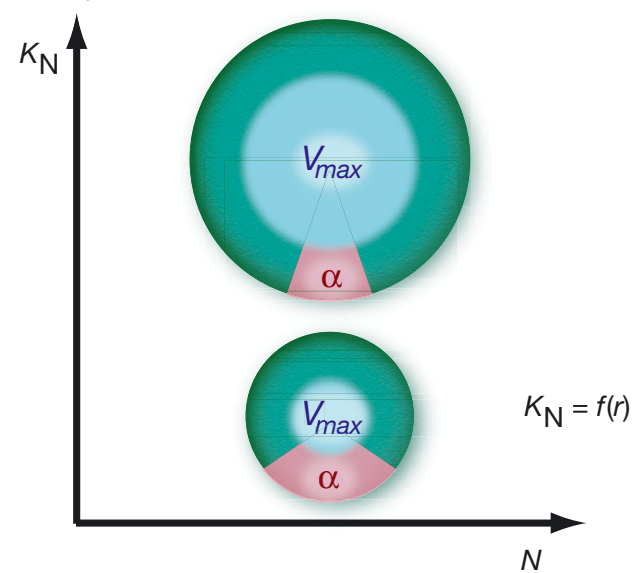

C) This study

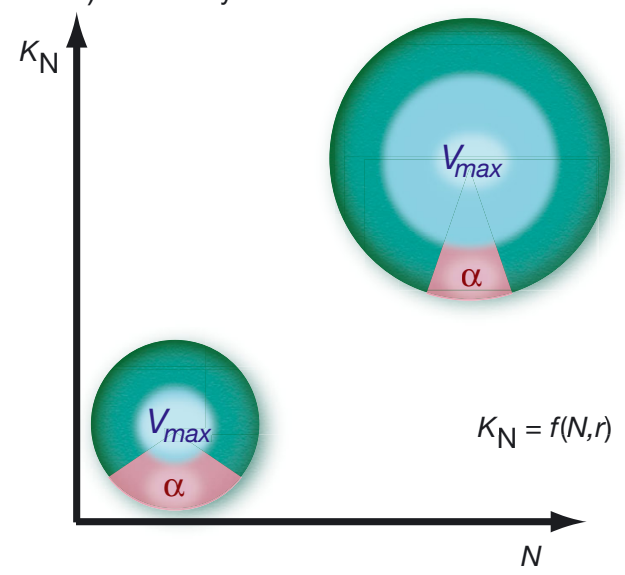

Fig. 1. Explanations for observed variations of half-saturation constants, $K_{\mathrm{N}}$, for nutrient uptake, shown in terms of the physiological traits. Sizes of components represent relative trait values: blue inner circles for $V_{\max }$ (relative allocation of intracellular resources to enzymes), pink section of outer circle for $\alpha$ (relative allocation of intracellular resources to nutrient porters), and green section of outer circles for cell sizes (expressed as radius, $r$ ). (A) $K_{\mathrm{N}}$ increases with ambient nutrient concentration $(N)$, irrespective of cell size, (B) $K_{\mathrm{N}}$ increases with cell size, irrespective of $N$, and (C) $K_{\mathrm{N}}$ increases with both cell size and $N$ showed that this same trade-off explains the observed increase of half-saturation constant for nitrate uptake, $K_{\mathrm{N}}$, with ambient nitrate concentration in the ocean.

Although recent studies (e.g. Ward et al. 2013, Wirtz 2013) have begun to address community structure in terms of size and physiological trade-offs, this has yet to be done specifically for nutrient uptake. Indeed, the optimal uptake (OU) trade-off (Pahlow 2005, Smith et al. 2009) has not considered the effects of size and therefore cannot explain the dependence of $K_{\mathrm{N}}$ on both cell size (Litchman et al. 2007, Edwards et al. 2012) and ambient nutrient concentration (Collos et al. 2005). Recent size-based models of nutrient uptake (Fiksen et al. 2013) do, however, provide a trait-based framework that links physiological and physical effects, i.e. extracellular diffusive limitation and temperature dependence, although not in terms of tradeoffs (Fig. 1B). Here we combine the OU trade-off for nutrient uptake and the size-based framework of Aksnes \& Cao (2011) to construct a new model that better explains the observed variability of $K_{\mathrm{N}}$ for marine phytoplankton (Fig. 1C).

\section{MODEL DESCRIPTION}

The trait-based framework proposed by Aksnes \& Cao (2011) consists of the following equation for nutrient uptake rate, $V$, as a function of the bulk (extra-cellular) nutrient concentration, $N$ (denoted $S$ by Aksnes \& Cao 2011):

where

$$
V(N)=\frac{B(N)}{2 A(N)}\left(1-\sqrt{1-\frac{4 A(N)}{[B(N)]^{2}}}\right)
$$

$$
\begin{gathered}
A(N)=\frac{h}{4 \pi D r N n}\left(1-\frac{\pi r p}{n s}\right) \\
B(N)=\frac{1}{\alpha N}+\frac{h}{n} \\
\alpha=4 \pi D r \frac{n s}{n s+\pi r(1-p)} \\
p=\frac{n s^{2}}{4 r^{2}}
\end{gathered}
$$

where $h$ is the handling time (time required to process 1 nutrient ion per uptake site), $D$ is the diffusivity of nutrient ions in the extracellular medium (water), $r$ is the cell radius, $n$ is the number of nutrient uptake sites (i.e. porters) per cell, $s$ is the radius of a single nutrient uptake site, and $p$ is the fraction of the cell surface covered by uptake sites. The factor $A(N)$ is the reciprocal of the product of the maximum encounter rate times the maximum uptake rate, and $B(N)$ is the sum 
of 2 resistances, namely the reciprocals of the timescales for encountering nutrient ions at the cell surface (first term) and handling time (second term). Here $\alpha$ is the affinity for nutrient uptake on a per cell basis, and the factor $\pi r(1-p)$ accounts for interactions between uptake sites (Zwanzig 1990). Table 1 presents the model parameters, their units, and assumed size-scalings. Aksnes \& Cao (2011) differentiate between apparent traits (affinity and half-saturation constants as typically measured) and inherent traits $(r$, $n, s$, and $h$ ) and make a theoretically sound case that the latter provide a mechanistic understanding of nutrient uptake kinetics. Moreover, they specifically suggest that trade-offs are better understood in terms of inherent rather than apparent traits.

Building on the model of Aksnes \& Cao (2011), Fiksen et al. (2013, p. 193) conclude that there is 'no mechanistic foundation for a trade-off conflict between the half-saturation coefficient and the maximum specific uptake rate'.

This conclusion results directly from their definition of handling time, $h$, as the time required for a single uptake site to process a single nutrient ion, so that the maximum uptake rate per cell is $V_{\max }=n / h$. They acknowledge that neither $h$ nor $n$ is constant and back-calculate their size-scalings based on observed allometries for $V_{\max }$ and half-saturation constants, under the assumption that $h$ and $n$ vary only with cell size. Thus, they predict that the half-saturation constant and the maximum uptake rate should both in- crease with the number of nutrient porters, $n$, so that no trade-off emerges (Fiksen et al. 2013, their Fig. 3C). This is surprising because mechanistic reasoning suggests that the benefits of intracellular resource allocation to enhance any given competitive ability are in general balanced by opportunity costs (Geider et al. 2009, Smith et al. 2011). Photo-acclimation is one well-known example of this principle, in which resources are allocated between light harvesting, carbon fixation, and biosynthesis (Wirtz \& Pahlow 2010, Talmy et al. 2013). The trade-off between nutrient affinity and maximum uptake rate is another example (Pahlow 2005, Smith et al. 2009).

In an attempt to reconcile these 2 different aspects, we combine the OU trade-off (Pahlow 2005, Smith et al. 2009) with the trait-based framework of Fiksen et al. (2013) and then examine the implications. Aksnes \& Egge (1991) advanced our understanding of nutrient uptake kinetics by deriving their equation explicitly at the cellular level, rather than at the level of individual enzymes for which the Michaelis-Menten equation was originally obtained. In the same spirit, we build on the notation of Aksnes \& Egge (1991) and Aksnes \& Cao (2011), by defining the handling time on a per cell basis, $h_{\mathrm{c}}$. Thus, the maximum uptake rate per cell is simply $V_{\max }=1 / h_{\mathrm{C}}$. Our overall goal is to apply the trait-based framework for nutrient uptake kinetics at the cellular level.

The definition of $h$ is critical, because the OU tradeoff is based on the allocation of resources to nutrient

Table 1. Parameters, their values (where fixed) and size-scalings (where assumed) for the nutrient uptake model. Potential minimum handling time and potential maximum number of porters were estimated from the size-scalings reported by Fiksen et al. (2013), assuming that the phytoplankton under culture conditions were adapted to high nutrient conditions, which implies allocation of resources heavily towards increasing maximum uptake rate, at the expense of reducing affinity (the fractional allocation of resources to nutrient porters $\left(f_{A}\right)=0.05$ was assumed for conversion via Eq. 2). Scaling slope: allometric scaling slope with cell radius $r$

\begin{tabular}{|c|c|c|c|c|}
\hline Paramete & r Value & Units & Description & $\begin{array}{l}\text { Scaling } \\
\text { slope }\end{array}$ \\
\hline$h$ & & s molecule ${ }^{-1}$ & Handling time per nutrient ion per porter & \\
\hline$n$ & & & No. of porters per cell & \\
\hline$n_{\max }^{\mathrm{ref}}$ & 2440 & & $\begin{array}{l}\text { Reference value of potential maximum no. of porters per cell, specified } \\
\text { for } r=r_{0}\end{array}$ & 2 \\
\hline$r_{0}$ & $6.2 \times 10^{-7}$ & $\mathrm{~m}$ & Reference cell radius & \\
\hline$h_{\mathrm{c}}$ & & s molecule ${ }^{-1}$ & Handling time per nutrient ion per cell & \\
\hline$h_{\mathrm{C}, \min }^{\mathrm{ref}}$ & $1.5 \times 10^{-5}$ & s molecule ${ }^{-1}$ & $\begin{array}{l}\text { Reference value of potential minimum handling time per nutrient ion } \\
\text { per cell, specified for } r=r_{0}\end{array}$ & -3 \\
\hline$D$ & $1.0 \times 10^{-9}$ & $\mathrm{~m}^{2} \mathrm{~s}^{-1}$ & Diffusivity of nutrient ions in extracellular water & \\
\hline$S$ & $1.0 \times 10^{-9}$ & $\mathrm{~m}$ & Radius of porter, i.e. uptake site & \\
\hline$N$ & & $\mathrm{M}^{-3}$ & Bulk extracellular nutrient concentration (no. of molecules per unit volume) & \\
\hline$\alpha$ & & $\mathrm{m}^{3} \mathrm{~s}^{-1}$ & Affinity (per cell) calculated using Eq. (1d) & \\
\hline$A(N)$ & & $\mathrm{s}^{2}$ molecule ${ }^{-2}$ & Term from Eq. (1b) & \\
\hline$B(N)$ & & s molecule ${ }^{-1}$ & Term from Eq. (1c) & \\
\hline$V$ & & molecule $\mathrm{s}^{-1}$ & Nutrient uptake rate (per cell) from Eq. (1a) & \\
\hline
\end{tabular}


porters (increasing $n$ and hence $\alpha$ ) vs. other enzymes (increasing $V_{\max }$ ). In this context, increasing $n$ (per cell) implies increasing handling time on a per cell basis, $h_{\mathrm{c}}$, because the number of rate-limiting enzymes (and hence $V_{\max }$ ) decreases as $n$ increases. Increasing $n$ necessarily implies also increasing $h$ on a per porter basis, because $h=n h_{\mathrm{C}} \cdot \underline{1}$ We express the OU trade-off in terms of $h_{\mathrm{c}}$ and $n$ as:

$$
\begin{aligned}
h_{\mathrm{c}} & =\frac{h_{\mathrm{c}, \min }}{1-f_{A}} \\
n & =f_{A} n_{\max }
\end{aligned}
$$

where $h_{c, \min }$ is the potential minimum handling time per cell, $n_{\max }$ is the potential maximum number of porters, and $f_{A}$ is the fractional $(0,1)$ allocation of resources to nutrient porters vs. whatever rate-limiting enzymes determine $h_{\mathrm{C}}$. Thus, as $f_{A}$ approaches 0 , $h_{\mathrm{C}}$ approaches $h_{\mathrm{c}, \mathrm{min}}$. Conversely as $f_{\mathrm{A}}$ approaches 1 , $n$ approaches $n_{\max }$ and $h_{\mathrm{c}}$ increases without bound, meaning that $V_{\max }$ approaches 0 . This physiologically explicit expression of the trade-off proposed in terms of $\alpha$ and $V_{\max }$ (Pahlow 2005) makes clear that there is no contradiction with the trait-based model of Aksnes \& Cao (2011).

The model of Aksnes \& Cao (2011) predicts that the following effective value of half-saturation constant ( $K_{Q_{\infty}}$ in their notation) should be observed by typical bulk-scale experiments (Fiksen et al. 2013):

$$
K_{\mathrm{N}}=\frac{\pi r(2-p)+n s}{8 h \pi r s D}
$$

This equation relates the underlying traits $(n, s$ and $r$ ) as well as the diffusivity $(D)$ to the observable $K_{\mathrm{N}}$. In order to consistently compare the model of Aksnes \& Cao (2011) and our model (as defined by Eqs. 1 and 2), we consider 2 size-scaling relationships, for potential minimum handling time and potential maximum number of sites (Table 1). First, we assume that $h_{\mathrm{c}, \min }$ decreases with increasing cell volume, i.e. scales as $r^{-3}$

$$
h_{\mathrm{c}, \min }=h_{\mathrm{c}, \min }^{\text {ref }}\left(\frac{r}{r_{0}}\right)^{-3}
$$

which is equivalent to assuming that the potential maximum of $V_{\max }$ increases with cell volume. The finding of Marañón et al. (2013) that $V_{\max }$ increases

\footnotetext{
${ }^{1}$ The relationship between the handling time per ion per porter, $h$, and the handling time per ion per cell, $h_{\mathrm{C}}$ as defined herein, is more obvious in terms of rates. The nutrient processing rate per porter, $r=1 / h$, and the overall rate per cell, $V_{\max }=1 / h_{\mathrm{c}}$. The overall rate per cell must equal the number of porters per cell, $n$, times the rate per porter: $V_{\max }=n r$. Substituting the above expressions for $V_{\max }$ and $r$ gives: $h=n h_{\mathrm{c}}$
}

proportional to cell volume does provide some basis for this assumed scaling, because their semi-continuous cultures reflect high-nutrient conditions (corresponding to low $f_{A}$ in our model framework), under which $V_{\max }$ should approach its potential maximum. However, in our model, the size-scaling of $V_{\max }$ itself depends on the ambient nutrient concentration through the allocation of intra-cellular resources $\left(f_{A}\right)$. Second, we assume that $n_{\max }$ increases with surface area, i.e. scales as $r^{2}$

$$
n_{\max }=n_{\max }^{\text {ref }}\left(\frac{r}{r_{0}}\right)^{2}
$$

which implies a fixed potential maximum fractional coverage of cellular surface area by porters, $p_{0}$ (as per Eq. 1e).

We numerically calculate the value of resource allocation, $f_{A}$, that maximizes nutrient uptake rate, $V$, for each given combination of nutrient concentration $(N)$ and cell size $(r)$. The optimization problem can be expressed as:

$$
\begin{aligned}
& \text { maximize }_{f_{\mathrm{A}}} V\left(f_{A} \mid N, r\right) \\
& \text { subject to } 0<f_{A}<1
\end{aligned}
$$

These optimal values of $f_{A}$ (Fig. 2A) are used to calculate the corresponding values of $n, h_{\mathrm{c}}$ and hence $h$, which are substituted into Eq. (3) to calculate the values of $K_{\mathrm{N}}$ for comparison against the observations (Fig. 2B) compiled from various ship-board incubation studies (Harrison et al. 1996, McCarthy et al. 1999, Collos et al. 2005).

\section{RESULTS AND DISCUSSION}

With the assumption of optimal resource allocation, the modeled relationships of $K_{\mathrm{N}}$ and ambient nitrate concentration compare well to a compilation of $K_{\mathrm{N}}$ values observed for natural communities from a wide variety of marine environments (Fig. 2B). Our model predicts that $K_{\mathrm{N}}$ scales with the square root of the ambient nitrate concentration for any given cell size (Fig. 2B), as for the size-independent OU kinetics (Smith et al. 2009). The cell size composition of the phytoplankton community corresponding to each observed $K_{\mathrm{N}}$ was not reported for these observations. However, the range of predicted $K_{\mathrm{N}}$ values for typical phytoplankton cell sizes in the ocean $(0.2$ to $100 \mu \mathrm{m})$ covers the observed range in the data.

Furthermore, the observed increase of $K_{\mathrm{N}}$ with ambient nitrate concentration for the data in Fig. 2B is steeper (log-log slope $=0.643[ \pm 0.045], \mathrm{r}^{2}=0.66$, $F=200, \mathrm{n}=103, \mathrm{p}<0.001)$ than our model's prediction (log-log slope $=0.5$ ) for any given cell size. This 
is consistent with the well-known notion that natural assemblages tend to have larger (smaller) cell sizes at higher (lower) ambient nutrient concentrations and that larger cells tend to have greater half-saturation
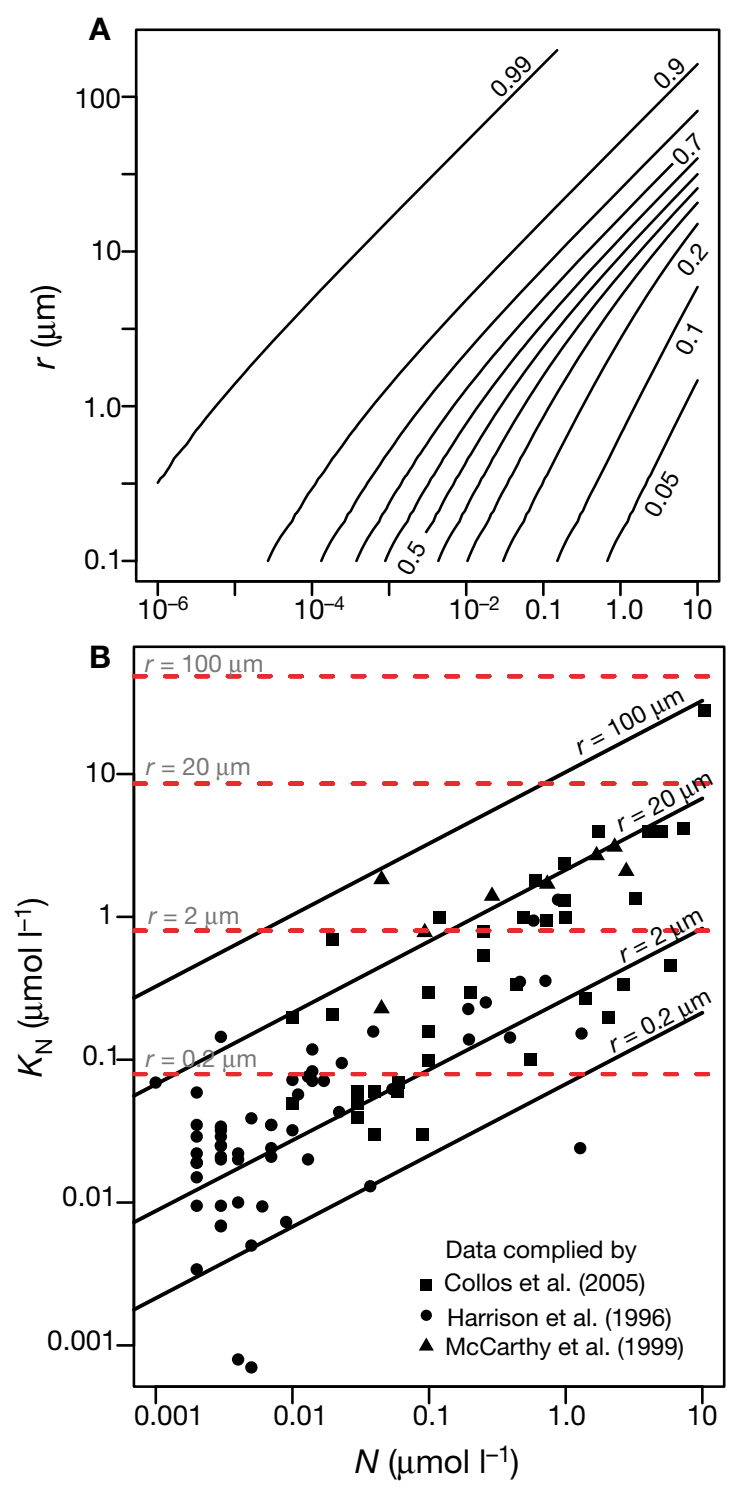

Fig. 2. (A) Contours of optimal values of intracellular resource allocation, $f_{\mathrm{A}}$, as a function of cell size (radius, $r$ ) and ambient nitrate concentration $(N)$. Here the values of $N$ used in the calculations (molecules $\mathrm{m}^{-3}$ ) were converted to $\left(\mu \mathrm{mol} \mathrm{l}^{-1}\right)$. (B) The resulting predicted pattern of half-saturation constants for nitrate uptake $\left(K_{\mathrm{N}}\right)$ versus $N$, for selected cell sizes covering the range of phytoplankton observed in the ocean, compared with data (symbols) compiled from a wide variety of marine environments. Solid lines show predicted values from the size-based model including the physiological trade-off, and dashed horizontal red lines show those from the trait-based model without this trade-off (Fiksen et al. 2013) constants (Litchman et al. 2007, Edwards et al. 2012). Conversely, an agreement of the predicted slope of $K_{\mathrm{N}}$ with ambient nitrate concentration for any given cell size with the overall slope of the observations would suggest a flaw in the model. The increase in dominant cell size with ambient nitrate concentration provides an explanation for why the square root dependence (log-log slope of 0.5 ) predicted by OU kinetics is consistently weaker than the empirical slope of $K_{\mathrm{N}}$ vs. ambient $N$ based on data sets from different oceanic regions (Fig. 2B herein, Collos et al. 2005, Smith et al. 2009, their Fig. 2).

Given the lack of concomitant observations of nutrient uptake kinetics and cell size distributions for natural assemblages of phytoplankton sampled for ship-board experiments, this semi-quantitative test of the model is the best that we can currently provide. However, Fig. 2B also highlights the contrast between our model's predictions and the size-scalings of Fiksen et al. (2013).

By contrast, the size-scalings derived from allometries based on laboratory experiments under the assumption of no trade-off (Fiksen et al. 2013) systematically over-estimate the half-saturation constants from field samples at low nutrient concentrations (Fig. 2B), even for the smallest realistic cell size $(0.2 \mu \mathrm{m})$. Given that large cells tend to become more dominant as nutrient concentration increases, their model, which assumes an increase of $K_{\mathrm{N}}$ with cell size, would therefore also imply an increase of $K_{\mathrm{N}}$ with ambient nutrient concentration. In order to explain the observations, their model would require a steeper decrease of $h$ with increasing size. The two models agree more closely at high nutrient concentrations, suggesting that their size-scaling relationships reflect a bias in the laboratory cultures towards high-nutrient concentrations and species that are easily cultured under those conditions.

By incorporating the physiological trade-off of OU kinetics into this framework, we have accounted for the dependence of nutrient uptake kinetics on both ambient nutrient concentration and cell size (Fig. 1). The resulting model reproduces the observed spread of $K_{\mathrm{N}}$ data for any nutrient concentration based on a realistic range of phytoplankton cell sizes. Our approach also captures the dependence of $K_{\mathrm{N}}$ on the nutrient concentration $N$. However, it remains possible that other physical factors such as temperature (Aksnes \& Cao 2011) may also contribute to determining the overall observed pattern. Future observational studies, particularly focusing on the relationships between size and physiology under ambient conditions would be particularly valuable for testing 
key assumptions of our model, such as the sizescalings of $h_{\mathrm{C}, \min }$ and $n_{\max }$.

Aksnes \& Cao (2011) and Fiksen et al. (2013) have provided a useful framework for connecting nutrient uptake kinetics to underlying biological and physical properties, i.e. inherent traits. Our results support their case that nutrient uptake kinetics are better understood and modeled in terms of inherent, rather than apparent, traits, and furthermore suggest a refinement of their framework. We propose that quantities varying as a function of physiological regulation (i.e. optimal resource allocation) are better considered as apparent, rather than inherent or intrinsic, trait values. Specifically, we find that the number of uptake sites, $n$, and the handling time on a per site basis, $h$, both vary with the allocation of intracellular resources, which is optimized for given environmental conditions. In the context of acclimation by a single species (Pahlow 2005, Smith \& Yamanaka 2007), $n$ and $h$ are therefore better considered apparent traits, and the potential maximum number of uptake sites per cell, $n_{\max }$ and the potential minimum handling time, $h_{\mathrm{c}, \min }$, that we introduced are the inherent traits. In the context of inter-specific differences, as shown in Fig. $2, n$ and $h$ (hence $f_{A}$ as well) represent inherent traits that differ among species, and $n_{\max }$ and $h_{\mathrm{c} \text {,min }}$ represent the intrinsic limits of their corresponding trait values. Such hyperparameters are precisely what are needed for modeling the biodiversity and adaptive capacity of communities by combining traits and trade-offs (Smith et al. 2014), as in the 'adaptive dynamics' approach (Wirtz \& Eckhardt 1996, Norberg et al. 2001, Merico et al. 2009).

Most theories for explaining community structure and biodiversity are based on the concept that organisms face trade-offs in their use of resources (Tilman 2000). Not surprisingly, trade-offs are key components in the recent generation of plankton ecosystem models that represent either multiple species (Follows et al. 2007, Hickman et al. 2010) or the whole community as a single adaptive entity (Wirtz \& Eckhardt 1996, Norberg et al. 2001, Merico et al. 2009). Analogous to the suggestion of Fiksen et al. (2013) that data should be interpreted in terms of inherent traits, we argue that progress in modeling is likely to come from accounting for and explicitly formulating trade-offs in terms of such inherent traits.

\section{LITERATURE CITED}

Aksnes DL, Cao FJ (2011) Inherent and apparent traits in microbial nutrient uptake. Mar Ecol Prog Ser 440:41-51
Aksnes DL, Egge JK (1991) A theoretical model for nutrient uptake in phytoplankton. Mar Ecol Prog Ser 70:65-72

Armstrong RA (2008) Nutrient uptake rate as a function of cell size and surface transporter density: a Michaelislike approximation to the model of Pasciak and Gavis. Deep-Sea Res I 55:1311-1317

Bonachela JA, Raghib M, Levin SA (2011) Dynamic model of flexible phytoplankton nutrient uptake. Proc Natl Acad Sci USA 108:20633-20638

$>$ Bruggeman J, Kooijman SALM (2007) A biodiversity-inspired approach to aquatic ecosystem modeling. Limnol Oceanogr 52:1533-1544

Chisholm SW (1992) Phytoplankton size. In: Falkowski PG, Woodhead AD (eds) Primary productivity and biogeochemical cycles in the sea. Plenum Press, New York, NY, p 213-237

Collos Y, Vaquer A, Souchu P (2005) Acclimation of nitrate uptake by phytoplankton to high substrate levels. J Phycol 41:466-478

$>$ Edwards KF, Thomas MK, Klausmeier CA, Litchman E (2012) Allometric scaling and taxonomic variation in nutrient utilization traits and maximum growth rate of phytoplankton. Limnol Oceanogr 57:554-566

Edwards KF, Litchman E, Klausmeier CA (2013) Functional traits explain phytoplankton community structure and seasonal dynamics in a marine ecosystem. Ecol Lett 16: $56-63$

Fiksen Ø, Follows MJ, Aksnes DL (2013) Trait-based models of nutrient uptake in microbes extend the MichaelisMenten framework. Limnol Oceanogr 58:193-202

Finkel VF, Beardall J, Flynn KJ, Quigg A, Rees TAV, Raven JA (2010) Phytoplankton in a changing world: cell size and elemental stoichiometry. J Plankton Res 32:119-137

Follows MJ, Dutkiewicz S, Grant S, Chisholm SW (2007) Emergent biogeography of microbial communities in a model ocean. Science 315:1843-1846

Geider RJ, Moore CM, Ross ON (2009) The role of costbenefit analysis in models of phytoplankton growth and acclimation. Plant Ecol Divers 2:165-178

> Harrison WG, Harris LR, Irwin DB (1996) The kinetics of nitrogen utilization in the oceanic mixed layer: nitrate and ammonium interactions at nanomolar concentrations. Limnol Oceanogr 41:16-32

Hickman AE, Dutkiewicz S, Williams RG, Follows MJ (2010) Modelling the effects of chromatic adaptation on phytoplankton community structure in the oligotrophic ocean. Mar Ecol Prog Ser 406:1-17

$>$ Kostadinov TS, Siegel DA, Maritorena S (2010) Global variability of phytoplankton functional types from space: assessment via the particle size distribution. Biogeosciences 7:3239-3257

> Li WKW (2002) Macroecological patterns of phytoplankton in the northwestern North Atlantic Ocean. Nature 419: 154-157

Litchman E, Klausmeier CA (2008) Trait-based community ecology of phytoplankton. Annu Rev Ecol Evol Syst 39: 615-639

- Litchman E, Klausmeier CA, Schofield OM, Falkowski PG (2007) The role of functional traits and trade-offs in structuring phytoplankton communities: scaling from cellular to ecosystem level. Ecol Lett 10:1170-1181

- Marañón E, Holligan PM, Barciela R, González N, Mouriño B, Pazó MJ, Varela M (2001) Patterns of phytoplankton size structure and productivity in contrasting open-ocean environments. Mar Ecol Prog Ser 216:43-56 
Marañón E, Cermeño P, Lopez-Sandoval DC, RodriguezRamos T and others (2013) Unimodal size scaling of phytoplankton growth and the size dependence of nutrient uptake and use. Ecol Lett 16:371-379

McCarthy JJ, Garside C, Nevins JL (1999) Nitrogen dynamics during the Arabian Sea Northeast Monsoon. DeepSea Res II 46:1623-1664

Merico A, Bruggeman J, Wirtz K (2009) A trait-based approach for downscaling complexity in plankton ecosystem models. Ecol Model 220:3001-3010

Norberg J, Swaney DP, Dushoff J, Lin J, Casagrandi R, Levin SA (2001) Phenotypic diversity and ecosystem functioning in changing environments: a theoretical framework. Proc Natl Acad Sci USA 98:11376-11381

Pahlow M (2005) Linking chlorophyll-nutrient dynamics to the Redfield N:C ratio with a model of optimal phytoplankton growth. Mar Ecol Prog Ser 287:33-43

Smith SL, Yamanaka Y (2007) Optimization-based model of multinutrient uptake kinetics. Limnol Oceanogr 52: 1545-1558

Smith SL, Pahlow M, Merico A, Wirtz KW (2011) Optimalitybased modeling of planktonic organisms. Limnol Oceanogr 56:2080-2094

Smith SL, Yamanaka Y, Pahlow M, Oschlies A (2009) Optimal uptake kinetics: physiological acclimation explains the observed pattern of nitrate uptake by phytoplankton

Editorial responsibility: Katherine Richardson, Copenhagen, Denmark in the ocean. Mar Ecol Prog Ser 384:1-12

Smith SL, Merico A, Wirtz KW, Pahlow M (2014) Leaving misleading legacies behind in plankton ecosystem modelling. J Plankton Res 36:6123-620

Talmy D, Blackford J, Hardman-Mountford NJ, Dumbrell AJ, Geider RJ (2013) An optimality model of photoadaptation in contrasting aquatic light regimes. Limnol Oceanogr 58:1802-1818

Tilman D (2000) Causes, consequences and ethics of biodiversity. Nature 405:208-211

Ward BA, Dutkiewicz S, Follows MJ (2013) Modelling spatial and temporal patterns in size-structured marine plankton communities: top-down and bottom-up controls. J Plankton Res 0:1-17

Wirtz KW (2013) Mechanistic origins of variability in phytoplankton dynamics. I. Niche formation revealed by a size-based model. Mar Biol 160:2319-2335

Wirtz KW, Eckhardt B (1996) Effective variables in ecosystem models with an application to phytoplankton succession. Ecol Model 92:33-54

Wirtz KW, Pahlow M (2010) Dynamic chlorophyll and nitrogen:carbon regulation in algae optimizes instantaneous growth rate. Mar Ecol Prog Ser 402:81-96

Zwanzig R (1990) Diffusion-controlled ligand-binding to spheres partially covered by receptors: an effective medium treatment. Proc Natl Acad Sci USA 87:5856-5857

Submitted: December 5, 2013; Accepted: June 4, 2014

Proofs received from author(s): August 22, 2014 